\title{
Structure-Activity Relationships of Heparin
}

\author{
INDEPENDENCE OF HEPARIN CHARGE DENSITY AND \\ ANTITHROMBIN-BINDING DOMAINS IN THROMBIN INHIBITION \\ BY ANTITHROMBIN AND HEPARIN COFACTOR II
}

RoBERT E. HURST and MAN-CHIU POON, Department of
Environmental Health Sciences, School of Public Health, Division of
Hematology/Oncology, Department of Medicine and Birmingham Veterans
Administration Medical Center, University of Alabama, Birmingham,
Alabama 35294

Michael J. Griffith, Division of Hematology, Department of Medicine, University of North Carolina, Chapel Hill, North Carolina 27514

\begin{abstract}
A B S T RACT To better understand how heparin structure affects its activity the relationships between the functional domains for inhibitor binding and charge density were investigated to determine how these domains affect heparin-mediated thrombin inhibition by two different heparin-dependent protease inhibitors, antithrombin (AT) and heparin cofactor II (HC II). A series of heparins, fractionated systematically by charge density, was further fractionated on antithrombin agarose to isolate more homogeneous subfractions that were either inactive or highly active with respect to thrombin inhibition by AT. With AT, the activities of the AT-active subfractions increased sharply with heparin charge density, while those with little or no affinity for AT were virtually inactive. In contrast, with HC II inhibitor, the activities of the heparins depended only upon their charge densities and were independent of AT affinity. At any given charge density, the heparin before fractionation by AT affinity and the fractions that were highly active and inactive with AT were all equally active with HC II. The two inhibitors also differed in their reactivity with heparan sulfate and dermatan sulfate. A charge-density effect with the subfractions having similar high affinity for AT demonstrates that charge density rep-
\end{abstract}

Address correspondence to Dr. Hurst. Dr. Hurst's current address is Department of Urology, University of Oklahoma Health Sciences Center, Oklahoma City, OK 73190. Dr. Poon's current address is Department of Medicine, The University of Calgary, Calgary, Alberta, Canada T2N 4N1.

Received for publication 31 March 1983 and in revised form 25 May 1983. resents a heparin functional domain that is independent of the AT-binding domain. The behavior of the AT-inactive heparins, being fully active with HC II, demonstrates the functional domain necessary for AT binding is not needed to produce HC II activity.

\section{INTRODUCTION}

The polysaccharide heparin catalyzes the reaction between antithrombin (AT) ${ }^{1}$ and several serine proteases, and between heparin cofactor II (HC II) (1) and thrombin. How structure affects activity is not generally well known, and only in the case of AT binding has a physical structure been identified as being responsible for a given function. A short sequence of defined structure (2) is necessary for AT-heparin binding and, hence, activity with AT $(3,4)$. Because additional properties such as molecular weight $(5,6)$, thrombin affinity (6-8), and charge density (9-12), can also markedly affect the activity of a heparin, the molecule must have multiple functional domains $(6,7)$.

In this paper we report investigations to determine whether heparin functional domains required for ac-

\footnotetext{
${ }^{1}$ Abbreviations used in this paper: AT, antithrombin II/ III, also heparin cofactor I; $C^{50 \%}$, concentration of glycosaminoglycan producing $50 \%$ thrombin inhibition under standard conditions; $C_{\mathrm{R}}{ }^{50 \%}$, relative concentrations of glycosaminoglycan and standard heparin producing $50 \%$ thrombin inhibition under standard conditions; HA heparin, heparin with high affinity for AT; HC II, heparin cofactor II; LA heparin, heparin with low affinity for AT; VLA heparin, heparin with very low affinity for AT: Z, heparin linear charge density.
} 
tivity with AT also are important for thrombin inhibition with HC II. Our approach was to systematically fractionate heparin to functionally separate the effects of heparin charge density and AT affinity. It was thereby possible to investigate the roles of such functional domains without physically isolating or identifying the structures involved. By comparing the roles of different functional domains with two different inhibitors, it was possible to determine which domains are specific to a given inhibitor and which are general features of the mechanism of heparin catalysis. By learning how functionally different heparins act with these two different inhibitors, it may be possible to better understand the complex activity of heterogeneous heparin.

\section{METHODS}

Heparin fractionation. Hog mucosal heparin (176 U. S. Pharmacopeia, units per milligram; Diosynth BV, Holland) was fractionated by two-phase partition $(10,12)$ to obtain heparin fractions, previously characterized in detail (9-12), that vary systematically in linear charge density, $Z$. The highly purified and more homogeneous fractions have similar molecular-weight distributions, with an average molecular weight of 15,000 , and contain no detectable dermatan sulfate or other contaminating glycosaminoglycans. Five of the nine fractions were further fractionated by AT-agarose affinity chromatography (4) (see Table I). The size distributions of the high affinity (HA) and very low affinity (VLA) subfractions of fraction $3(Z=2.71)$ were compared by passing each through a column $(0.8 \mathrm{~cm} \times 60 \mathrm{~cm})$ of $6 \%$ agarose (4-0.5 m; Bio-Rad Laboratories, Richmond, CA) and 0.5-ml fractions were collected and analyzed for uronate as described previously (9).

Inhibitor, protease, and polysaccharide preparation. Human $\alpha$-thrombin was prepared as described previously (13). Human AT isolated by heparin-agarose affinity chro-

TABLE I

Physical Characteristics and Distribution by AT Affinity of Charge-Density Fractionated Heparins

\begin{tabular}{ccccc}
\hline & & \multicolumn{3}{c}{ Percent eluting with } \\
\cline { 3 - 5 } Fraction & $\begin{array}{c}\text { Charge } \\
\text { density } 1\end{array}$ & $\begin{array}{c}0.05 \mathrm{M} \mathrm{NaCl} \\
(\mathrm{VLA})\end{array}$ & $\begin{array}{c}0.30 \mathrm{M} \mathrm{NaCl} \\
(\mathrm{LA})\end{array}$ & $\begin{array}{c}3.0 \mathrm{M} \mathrm{NaCl} \\
(\mathrm{HA})\end{array}$ \\
\hline \multirow{2}{*}{1} & 2.48 & 70 & 9 & 21 \\
2 & 2.64 & 60 & 11 & 29 \\
3 & 2.71 & $52 \S$ & 15 & $33 \S$ \\
4 & 2.73 & 47 & 14 & 39 \\
5 & 2.85 & 43 & 21 & 36 \\
\hline
\end{tabular}

- $1 \mathrm{mg}$ heparin was fractionated on 6.5-ml AT-agarose column by stepwise elution with two bed volumes of the indicated concentration of $\mathrm{NaCl}$ in $0.05 \mathrm{M}$ Tris, $\mathrm{pH}$ 7.4.

$\ddagger$ Measured by acridine orange titration (see reference 13).

$\$$ The modes of the size distributions of these fractions were essentially indistinguishable even though some enrichment of the HA fraction in larger chains and of the VLA fraction in smaller chains was evident. matography was a gift from Dr. Michael Blackburn. Human HC II was isolated by a modification of the procedure of Tollefson (1) using heparin-agarose affinity chromatography and DEAE-cellulose anion-exchange chromatography. Heparan sulfate, isolated from beef-lung byproducts, was obtained from Dr. J. A. Cifonelli. Dermatan sulfate was obtained from Miles Laboratories, Inc. (Elkhart, IN) and was treated with nitrous acid to remove trace heparin contaminants (14).

Heparin activity determination. Thrombin inhibition was assayed in a two-stage amidolytic assay. AT (75 $\mathrm{nM})$ or HC II (150 nM), thrombin (36 nM), and heparin (total volume of $0.3 \mathrm{ml}$ in $0.05 \mathrm{M}$ Tris, pH 7.5, $0.15 \mathrm{M} \mathrm{NaCl}, 1.0 \mathrm{mg} /$ $\mathrm{ml}$ bovine serum albumin) were incubated at $37^{\circ} \mathrm{C}$ for 60 $\mathrm{s}$ in a small polyethylene vial. The thrombin remaining was determined by transferring $0.2 \mathrm{ml}$ to a disposable cuvette (Sarstedt, Inc., Princeton, NJ) containing $0.55 \mathrm{ml}$ of $0.05 \mathrm{M}$ Tris, $\mathrm{pH} 8.3,0.15 \mathrm{M} \mathrm{NaCl}$, and $1.65 \times 10^{-4} \mathrm{M}$ of the thrombin substrate $N$ - $\alpha$-p-tosyl-glycine-proline-arginine- $p$-nitroanilide (Chromozym TH; Boehringer Mannheim Biochemicals, Indianapolis, IN). Substrate hydrolysis at $37^{\circ} \mathrm{C}$ was monitored by absorbance at $405 \mathrm{~nm}$. Five concentrations of each heparin, chosen to produce $10-90 \%$ thrombin inhibition, were assayed. With the standard heparin (Diosynth $\mathrm{BV}$ ), these ranged from $0.75 \mathrm{nM}$ uronate to $3.0 \mathrm{nM}$ uronate with AT and 4.0 to $9.0 \mathrm{nM}$ with HC II, heparin concentration, $C$, expressed in terms of uronate concentration. For each heparin concentration the fractional thrombin inhibition, $F$, was calculated as $\left(\Delta A_{0}-\Delta A_{h}\right) / \Delta A_{0}$, where $\Delta A$ is the absorbance change per minute, subscript 0 being without heparin (but with inhibitor and thrombin), and subscript $h$ being with heparin. Double-reciprocal plots of $1 / F$ vs. $1 / C$, were linear in the range $0.1<F<0.9$. The concentrations producing $F=0.5\left(C^{50 \%}\right)$ were then interpolated from leastsquares-fitted straight lines. The term $C_{\mathrm{R}}{ }^{50 \%}$ denotes $C^{50 \%}$ (test heparin) $/ C^{50 \%}$ (standard heparin), both measurements made under the same conditions of time, temperature, and protein concentrations. The relative standard deviation of replicate measurements of $C_{\mathrm{R}}{ }^{50 \%}$ is $\pm 8 \%$. To have larger numbers associated with more active heparin, we have elected to equate activity with the reciprocal of $C_{\mathrm{R}}{ }^{50 \%}$.

\section{RESULTS}

The activities of the charge-density fractions and their AT-affinity subfractions with AT and HC II are shown in Fig. 1 as a function of $Z^{2}$. The AT-mediated or HC II-mediated activities $\left(1 / C_{R}{ }^{50 \%}\right)$ of any given chargedensity fractionated heparin before affinity fractionation are essentially indistinguishable and can be described by the same linear function of $Z^{2}$. Note that, at a given concentration of thrombin and inhibitor, $C^{50 \%}$ was higher with the HC II system. However, the difference in absolute concentrations is equally manifested with both standard and test heparin, thereby giving identical values of $C_{\mathrm{R}}{ }^{50 \%}$. As expected, after fractionation by AT affinity, with AT, the HA fractions were much more active than were the original fractions while the VLA fractions were virtually inactive. However the activities of the HA fractions with AT still depended upon $Z^{2}$, and the slope of the line is the same as that observed before fractionation. Moreover, the slope is independent of the nature of the inhibitor, 


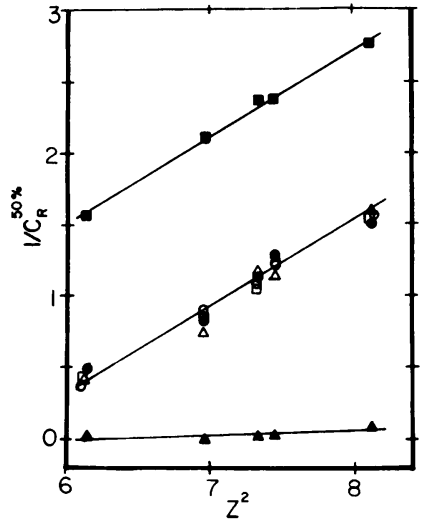

Figure 1 Activities $\left(1 / C_{\mathrm{R}}{ }^{50 \%}\right)$ of heparins in producing thrombin inhibition with AT and $\mathrm{HC}$ II as a function of the square of charge density $\left(Z^{2}\right)$. Activities with AT are shown using filled symbols and with HC II using open symbols. $\bigcirc \bullet$, heparins before affinity fractionation; $\square \mathbf{Q}$, HA heparins; $\Delta \Delta$, VLA heparins.

with HC II and AT giving the same slope. Finally, fractionation by AT affinity did not affect the activity of charge-density fractionated heparins in the HC II system in that HA and VLA heparins, which were highly active and inactive, respectively, with AT, were equally active with HC II. Moreover, for each charge density, the activities with HC II before and after ATaffinity fractionation are indistinguishable.

The two inhibitors also differed in their patterns of reactivity with heparan sulfate and dermatan sulfate. As shown in Table II, heparin-free dermatan sulfate, which is somewhat active with HC II $(\sim 1 / 200$ th as active as the standard heparin), is virtually inactive with AT. Heparan sulfate showed a very small amount of activity with AT, but none with HC II. The chondroitin sulfate was inactive with either inhibitor.

\section{DISCUSSION}

Heparin is not only a complex mixture of different glycosaminoglycan chains (12), but individual chains

TABLE II

Relative Concentrations of Glycosaminoglycan Required to Produce 50\% Thrombin Inhibition with AT and HC-II Inhibitors

\begin{tabular}{lcc}
\hline & \multicolumn{2}{c}{ Inhibitor } \\
\cline { 2 - 3 } \multicolumn{1}{c}{ Glycosaminoglycan } & AT & HC II \\
\hline Standard heparin & $1^{\circ}$ & 4 \\
Heparan sulfate & 2,600 & $>8,000 \ddagger$ \\
Dermatan sulfate & 5,000 & 800 \\
Chondroitin 4-sulfate & $>6,000 \ddagger$ & $>8,000 \ddagger$ \\
\hline
\end{tabular}

- Absolute concentration is $1.4 \mathrm{nM}$.

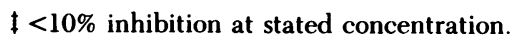

also seem to have multiple functional domains $(6,7)$, some of which have been identified with structural domains for inhibitor and protease binding $(2,6-8)$. In this study, systematically fractionated heparins were used to functionally isolate the effects of the charge density and AT-affinity domains. The twophase partition method is insensitive to molecular weight for molecular weights $>10,000$ and separates heparins according to charge density (9). The fractions were then each fractionated by AT affinity into HA and VLA subfractions of the same charge density. In this manner we produced three series (the HA, low affinity [LA], and VLA heparins) of much more homogeneous heparins with properties that were systematically related and that could be used to functionally isolate the AT affinity and charge-density effects.

Except the VLA heparins, which are inactive with AT, the activities of the heparins with both inhibitors show the same correlation with charge density. That such a charge-density effect is seen at all with the HA heparins is strong, direct evidence that, as predicted earlier from indirect evidence (10), the correlation with $Z^{2}$ arises from a domain that is independent of the AT-binding domain on the heparin. Moreover, the slope of the lines, which is a measure of the change in activity with $Z^{2}$, is insensitive to the nature of the inhibitor and is not changed by AT-affinity fractionation. Thus, the charge-density domain apparently acts similarly with these two different inhibitors, which suggests that the mechanism of the charge-density effect may not involve the inhibitor. One explanation is that charge density manifests its effect through the protease-binding domain (6-8).

Although $Z^{2}$ affects activity with the two different inhibitors in the same way, HC II does not appear to require the oligosaccharide-binding sequence required by AT. This is shown clearly by the failure of fractionation by AT affinity to alter in any way the activity with HC II. In other words, separating heparins into fractions such that the chains in one fraction all contain at least one complete AT-binding site (HA heparins), and the other in which none of the chains contain such a site (VLA heparin), did not alter the activity with HC II with respect to the unfractionated heparin of the same charge density but heterogeneous in AT affinity. Thus, we conclude that if a specific oligosaccharide sequence is required for heparin binding to HC II inhibitor, as a recent report suggests (15), it must be different from that required for antithrombin binding. More detailed structural work will be required to determine if a specific oligosaccharide sequence is required for $\mathrm{HC}$ II binding and, if such exists, whether it overlaps with or is completely independent of the AT-binding oligosaccharide sequence.

One of the difficulties in studies involving heparin fractionation is that many techniques will separate 
heparin by more than one structural variable at a time. For example, molecular-weight fractionation leads to concomitant fractionation by $\mathrm{AT}$ affinity and vice versa (5). It is interesting that $\mathrm{AT}$-affinity fractionation of charge-density fractionated heparins apparently produces much less concomitant molecular-weight fractionation than was observed for heparins that were heterogeneous in charge density (6). Our data suggest that possibly confounding concomitant fractionation may occur when charge-density heterogeneous heparin is fractionated by AT affinity in that the HA fraction will be selectively enriched in more highly charged heparins (see Table I). Tollefson and co-workers (1) showed that the HC-II activity of an HA fraction of heparin was higher than that of the heparin prior to AT-affinity fractionation, which led them to speculate the HC II and AT required the same oligosaccharide-binding sequence. However, the selective enrichment of the HA heparin with more highly charged chains, which as we have shown are more active with HC II, could account for their observation. It is thus critically important that individual structural variables, including charge density, molecular weight, and inhibitor affinity be each controlled.

Under certain circumstances, the contribution of thrombin inhibition by HC II may be quite important, even though its concentration in plasma is lower than that of AT (1) and requires higher concentrations of heparin to achieve a given level of activity. Heparin anticoagulant activity in AT-deficient plasma (16), or the unexpectedly high anticoagulant activities of heparins with low charge densities (11), may well at least in part, reflect HC II activity of AT-inactive heparins, while the small, but significant, anticoagulant activity of heparin-free dermatan sulfate (14) may reflect the activity dermatan sulfate has with HC II. The results also suggest that either heparin-free dermatan sulfate or VLA heparins might be useful for measuring HC II activity in plasma.

In summary, we found that heparin contains a charge-density domain that not only functioned independently of the AT-binding domain, but appeared to function identically with two completely different inhibitors. We also found that the sequence required for AT binding is not required for HC II activity. The use of systematically fractionated heparins, such as we and others (5-7) have used, offers an inviting path to eventually understanding the complex problem of relating heparin structure and activity.

\section{ACKNOWLEDGMENTS}

We wish to thank Dr. Blackburn (Louisiana State University Medical Center), Dr. G. W. K. van Dedem (Diosynth BV), and Dr. J. A. Cifonelli (University of Chicago) for their generous gifts of material.

This work was supported in part by U. S. Public Health
Service grants HL 24509 and HL 26309, and Veterans Administration Project MRIS 7133.

\section{REFERENCES}

1. Tollefsen, D. M., D. W. Majerus, and M. K. Blank. 1982. Purification and properties of a heparin-dependent inhibitor of thrombin in human plasma. J. Biol. Chem. 257:2162-2169.

2. Lindahl, U., G. Bäckstrom, L. Thunberg, and I. G. Leder. 1980. Evidence for a 3-O-sulfated D-glycosamine residue in the antithrombin-binding sequence of heparin. Proc. Natl. Acad. Sci. USA. 77:6551-6555.

3. Lam, L. H., J. E. Silbert, and R. D. Rosenberg. 1976. The separation of active and inactive forms of heparin. Biochem. Biophys. Res. Comm. 69:570-577.

4. Höök, M., I. Björk, J. Hopwood, and U. Lindahl. 1976. Anticoagulant activity of heparin: separation of highactivity and low-activity heparin species by affinity chromatography on immobilized antithrombin. FEBS (Fed. Eur. Biochem. Soc.) Lett. 66:90-93.

5. Danielsson, A., and I. Björk. 1981. Binding to antithrombin of heparin fractions with different molecular weights. Biochem. J. 193:427-433.

6. Laurent, T. C., A. Tengblad, L. Thunberg, M. Höök, and $U$. Lindahl. 1978. On the molecular weight dependence of the anticoagulant activity of heparin. $J$. Biochem. 175:691-701.

7. Oosta, G. M., W. T. Gardner, D. L. Beeler, and R. E. Rosenberg. 1981. Multiple functional domains of the heparin molecule. Proc. Natl. Acad. Sci. USA. 78:829833.

8. Griffith, M. J. 1979. Kinetic analysis of the heparin-enhanced antithrombin $111 /$ thrombin reaction rate enhancement by heparin-thrombin association. J. Biol. Chem. 254: 12034-12049.

9. Hurst, R. E., J. M. Menter, S. S. West, J. M. Settine, and E. H. Coyne. 1979. Structural basis for the anticoagulant activity of heparin. I. Relationship to the number of charged groups. Biochemistry. 18:4283-4287.

10. Menter, J. M., R. E. Hurst, D. A. Corliss, S. S. West, and E. W. Abrahamson. 1979. Structural basis for the anticoagulant activity of heparin. II. Relationship of anticoagulant activity to the thermodynamics and fluorescence fading kinetics of acridine orange-heparin complexes. Biochemistry. 18:4288-4292.

11. Hurst, R. E., G. W. K. van Dedem, and J. M. Settine. 1981. Biophysical characteristics of anionic density-fractionated heparins in relation to potencies in anticoagulant and thrombin-inhibition assays. Thromb. Res. 22:633-643.

12. Hurst, R. E., J. M. Settine, M.-C. Poon, and A. Lurie. 1982. Heterogeneity in the composition of commercial heparins: comparison of anticoagulant activities and biochemical compositions of anionic density-fractionated heparins. Thromb. Res. 25:255-265.

13. Lundblad, R. L., H. S. Kingdon, and K. G. Mann. 1976. Thrombin. Methods Enzymol. 45:151-176.

14. Kindness, G., W. F. Long, and F. B. Williamson. 1981. The anticoagulant activity of dermatan sulphates: evidence against the involvement of antithrombin III. $\mathrm{Br}$. J. Pharmacol. 72:81-88.

15. Griffith, M. J., and G. A. Marbet. 1983. Dermatan sulfate and heparin can be fractionated by affinity for heparin cofactor II. Biochem. Biophys. Res. Comm. 112:663670.

16. Holmer, E., G. Soderstrom, and L.-O. Andersson. 1980. Properties of antithrombin III depleted plasma I. Effect of heparin. Thromb. Res. 17:113-124. 